\title{
Synthesis of CdS and CdSe Nanocrystallites Using a Novel Single-Molecule Precursors Approach
}

\author{
Tito Trindade and Paul O'Brien* \\ Department of Chemistry Imperial College of Science, Technology and Medicine, \\ London SW7 2AY, U.K. \\ Xiao-mei Zhang
}

The Interdisciplinary Research Centre for Semi conductor Materials, The Blackett Laboratory, Imperial College of Science, Technol ogy and Medicine, London SW7 2BZ, U.K.

Received J uly 8, 1996. Revised Manuscript Received September 20, $1996^{\otimes}$

\begin{abstract}
The synthesis of CdS and CdSe nanocrystallites using the thermolysis of several dithioor disel enocarbamato complexes of cadmium in trioctyl phosphine oxide (TOPO) is reported. The nanodispersed materials obtained show quantum size effects in their optical spectra and exhibit near band-edge luminescence. The influence of experimental parameters on the properties of the nanocrystallites is discussed. HRTEM images of these materials show well-defined, crystalline nanosized particles. Standard size fractionation procedures can be performed in order to narrow the size dispersion of the samples. The TOPO-capped CdS and CdSe nanocrystallites and simple organic bridging ligands, such as 2,2'-bipyrimidine, are used as the starting materials for the preparation of novel nanocomposites. The optical properties shown by these new nanocomposites are compared with those of the starting nanodispersed materials.
\end{abstract}

\section{Introduction}

Nanocrystalline semiconductors have electronic properties intermediate between those of macrocrystalline solids and molecular entities. The properties of such materials are intriguing and have formed the subject of intense research in recent years. ${ }^{1-9}$ One interesting possibility is the use of particle size as a control parameter in order to tailor bandgap, which provides a novel approach to the development of materials for device applications; several uses are anticipated, including as photocatalysts, 10,11 light-emitting diodes, ${ }^{12,13}$ and in el ectrochemical cells. ${ }^{1,6,7}$

Nanocrystallites have been prepared by a wide range of synthetic methods. ${ }^{1-7}$ Many approaches involve aqueous solutions and the classical techniques of colloidal chemistry. However, for some technological important semiconductors, e.g., metal chalcogenides, such synthetic methodol ogies have limitations including

* To whom correspondence should be addressed. E-mail: p.obrien@ ic.ac.uk.

${ }^{\otimes}$ Abstract published in AdvanceACS Abstracts, December 1, 1996.

(1) Duonghong, D.; Ramsden, J .; Gratzell, M. J . Am. Chem. Soc. 1982, 104, 2977.

(2) Rossetti, R.; Ellison, J . L.; Gibson, J . M.; Brus, L. E. J . Chem. Phys. 1984, 80, 4464.

(3) Henglein, A. Chem. Rev. 1989, 89, 1861.

(4) Steigerwald, M. L.; Brus, L. E. Acc. Chem. Res. 1990, 23, 183.

(5) Wang, Y.; Herron, N. J . Phys. Chem. 1991, 95, 525.

(6) Weller, H. Adv. Mater. 1993, 5, 88.

(7) Hagfeldt, A.; Gratzell, M. Chem. Rev. 1995, 95, 49.

(8) Brus, L. E.J . Chem. Phys. 1984, 80, 4403.

(9) Brus, L. J . Phys. Chem. 1986, 90, 2555.

(10) Hoffman, A. J .; Yee, H.; Mills, G.; Hoffmann, M. R. J . Phys. Chem. 1992, 96, 5540.

(11) Hoffman, A. J .; Mills, G.; Yee, H.; Hoffmann, M. R. J . Phys. Chem. 1992, 96, 5546

(12) Colvin, V. L.; Schlamp, M. C.; Alivisatos, A. P. Nature 1994, $370,354$.

(13) Hakimi, F.; Bawendi, M. G.; Tumminelli, R.; Haavisto, J . R. U.S. Patent 5,260,957, 1993. the use of noxious compounds, such as $\mathrm{H}_{2} \mathrm{Se}$ or $\mathrm{H}_{2} \mathrm{~S}$, and a potential for the adventitious incorporation of oxide. We have been interested in the use of molecular compounds, containing both elements of the semiconductor, to produce the final nanodispersed material. ${ }^{14,15}$ In preliminary work high-quality CdSe nanocrystallites were obtained ${ }^{14}$ by the thermolysis, in hot tri-n-octylphosphine oxide (TOPO) as dispersing medium, of the single molecule precursor $\left[\mathrm{CH}_{3} \mathrm{CdSe}_{2} \mathrm{CN}\left(\mathrm{C}_{2} \mathrm{H}_{5}\right)_{2}\right]_{2}$. This synthetic approach is an extension of the TOPO method developed by Murray et al. ${ }^{16}$ which has been used to produce various II NI semiconductor nanoorystallites. ${ }^{16-19}$ One obvious advantage in using the single-source approach is that hazardous and volatile compounds such as $\left(\mathrm{CH}_{3}\right)_{2} \mathrm{Cd}$ are not involved in the high-temperatures procedures $\left(120-300{ }^{\circ} \mathrm{C}\right)$ used to produce the semiconductor material. Another, more ambitious possibility is that the method opens a way of obtaining a wide range of nanodispersed semiconductors with specific properties by tailoring the precursor. Such effects have been observed in the preparation of monodispersed micrometric inorganic colloids ${ }^{20-22}$ and in biomineralization processes, ${ }^{23}$ where it was found that a precipitate

(14) Trindade, T.; O'Brien, P. Adv. Mater. 1996, 8, 161.

(15) Trindade, T.; O'Brien, P. J . Mater. Chem. 1996, 6, 343.

(16) Murray, C. B.; Norris, D. J .; Bawendi, M. G. J. Am. Chem. Soc. 1993, 115, 8706.

(17) Bowen Katari, J . E.; Colvin, V. L.; Alivisatos, A. P. J . Phys. Chem. 1994, 98, 4109 .

(18) Dabbousi, B. O.: Bawendi, M. G.; Onitsuka, O.; Rubner, M. F. Appl. Phys. Lett. 1995, 66, 1317.

(19) Murray, C. B.; Kagan, C. R.; Bawendi, M. G. Science 1995, $270,1335$.

(20) Matijevic, E. Langmuir 1986, 2, 12

(21) Hamada, S.; Kudo, Y.; Okada, J .; Kano, H.J . Colloid Interface Sci. 1987, 118, 356.

(22) Trindade, T.; Pedrosa de J esus, J . D.; O'Brien, P. J . Mater. Chem. 1994, 4, 1611. 
can have morphological properties dependent on the chemical nature of the soluable precursors.

This paper describes a detailed study of the use of dithio- or diselenocarbamates of cadmium to produce $\mathrm{CdS}$ and CdSe nanocrystallites. The nanoparticulates can be used as starting compounds for the preparation of new materials. Organic ligands such as 2,2'-bipyrimidine (bpm) can be used to assemble the CdS or CdSe nanoparticulates into composites. The optical properties of these materials have been evaluated and are discussed in relation to those of the starting nanodispersed materials.

\section{Experimental Section}

2.1. Chemicals. $\mathrm{CdCl}_{2}, 99+\%$ (Aldrich); $\mathrm{NaS}_{2} \mathrm{CN}\left(\mathrm{C}_{2} \mathrm{H}_{5}\right)_{2}$. $3 \mathrm{H}_{2} \mathrm{O}, 98 \%$ (Aldrich); $\mathrm{CH}_{2} \mathrm{Cl}_{2} 99 \%$ (BDH); diethylamine, $98 \%$ (Aldrich); petroleum ether $40-60{ }^{\circ} \mathrm{C}(\mathrm{BDH})$; toluene (BDH); absolute methanol (BDH); diethyl ether (BDH); magnesium turnings (BDH); 1-bromo-2,2-dimethyl propane, 98\% (Aldrich); $\left(\mathrm{CH}_{3}\right)_{2} \mathrm{Cd}$ (E pichem); tri-n-octyl phosphine oxide, 90\% (Aldrich); tri-n-octylphosphine, 95\% (Aldrich); pyridine 99+\% (Aldrich); 2,2'-bipyrimidine, 96\% (Lancaster); pyrazine, 99+ \% (Lancaster); 4,4'-dipyridyl, $98 \%$ (Aldrich).

All the chemicals were used as received except TOPO, which was purified using the literature method. ${ }^{24}$ The solvents used for air-sensitive chemistry were distilled, deoxygenated under a nitrogen flow and stored over molecular sieves (type $4 \AA$, BDH) before use.

2.2. Syntheses. Singlemolecule precursors: $\left[\mathrm{Cd}\left\{\mathrm{S}_{2} \mathrm{CN}\right.\right.$ $\left.\left(\mathrm{C}_{2} \mathrm{H}_{5}\right)_{2}\right\}_{2} \mathrm{~J}_{2}$ was prepared from stoichiometric amounts of aqueous $\left(0.1 \mathrm{~mol} \mathrm{dm}^{-3}\right)$ solutions of $\mathrm{NaS}_{2} \mathrm{CN}\left(\mathrm{C}_{2} \mathrm{H}_{5}\right)_{2}$ and $\mathrm{CdCl}_{2}$. The white product obtained was filtered and washed with deionized water and recrystal lized from hot dichloromethane.

$\left[\mathrm{Cd}\left\{\mathrm{Se}_{2} \mathrm{CN}\left(\mathrm{C}_{2} \mathrm{H}_{5}\right)_{2}\right\}_{2}\right]_{2}$ was prepared ${ }^{25}$ by reacting $\mathrm{N}, \mathrm{N}-$ diethyldiselenocarbamate, as the diethylammonium salt, with an aqueous solution containing a stoichiometric amount of $\mathrm{CdCl}_{2}$. The yellow product obtained was filtered and washed with deionized water.

$\left[\mathrm{RCdE}_{2} \mathrm{CN}\left(\mathrm{C}_{2} \mathrm{H}_{5}\right)_{2}\right]_{2}, \mathrm{R}=\mathrm{Me}$ (methyl), $\mathrm{Np}$ (neopentyl) and $\mathrm{E}=\mathrm{S}$, Se: all these compounds were synthesized using standard Schlenk techniques in a nitrogen atmosphere. Typically, $\left[\mathrm{CH}_{3} \mathrm{CdS}_{2} \mathrm{CN}\left(\mathrm{C}_{2} \mathrm{H}_{5}\right)_{2}\right]_{2}$ was synthesized by the comproportionation reaction ${ }^{26}$ of $\left[\mathrm{Cd}\left\{\mathrm{S}_{2} \mathrm{CN}\left(\mathrm{C}_{2} \mathrm{H}_{5}\right)_{2}\right\}_{2}\right]_{2}$ and $\left(\mathrm{CH}_{3}\right)_{2} \mathrm{Cd}$ in toluene, at room temperature, over $2 \mathrm{~h}$. [ $\mathrm{NpCdS}_{2} \mathrm{CN}$ $\left.\left(\mathrm{C}_{2} \mathrm{H}_{5}\right)_{2}\right]_{2}$ was obtained in the same way using the metal neopentyl. The $\mathrm{Np}_{2} \mathrm{Cd}$ was prepared by reacting $\mathrm{Cdl}_{2}$ and the Grignard reagent $\mathrm{NpMgBr}$ in ether using the literature procedure. ${ }^{27}$ The selenium compounds were synthesized in a similar way using the analogous compounds. All the precursors used were identified by ${ }^{1} \mathrm{H}$ NMR spectroscopy and melting point measurements. ${ }^{25,26}$

CdS and CdSenanocrystallites: The nanodispersed materials were synthesized by injection of a solution of the singlemolecule precursor ( $\left[\mathrm{MeCdE}{ }_{2} \mathrm{CN}\left(\mathrm{C}_{2} \mathrm{H}_{5}\right)_{2}\right]_{2}$ unless otherwise indicated) into hot TOPO using standard Schlenk techniques and a nitrogen atmosphere. Typically $0.5 \mathrm{mmol}$ of $\left[\mathrm{MeCdS}_{2^{-}}\right.$ $\left.\mathrm{CN}\left(\mathrm{C}_{2} \mathrm{H}_{5}\right)_{2}\right]_{2}$ was mixed in $10 \mathrm{~mL}$ of TOP, and the mixture formed was filtered and then injected into $30 \mathrm{~g}$ of TOPO at $250^{\circ} \mathrm{C}$; the solution was then heated for $30 \mathrm{~min}$. The yellow solution which formed was allowed to cool to $75^{\circ} \mathrm{C}$, after which a large excess of dry $\mathrm{MeOH}$ was added. A flocculate of $\mathrm{CdS}$ formed which was isolated by centrifugation and redispersed in toluene; any insoluble material (poorly capped and large

(23) Biomineralization; Mann, S., Webb, J ., Williams, R. J . P., Eds.; VCH: Weinheim, 1989.

(24) Zingaro, R. A.; White, J . C. J . Inorg. Nuclear Chem. 1960, 12, 315.

(25) Hursthouse, M. B.; Azad Malik, M.; Motevalli, M.; O'Brien, P. Polyhedron 1992, 11, 45.

(26) Hursthouse, M. B.; Azad Malik, M.; Motevalli, M.; O'Brien, P. Organometallics 1991, 10, 730.

(27) O'Brien, P.; Walsh, J. R.; J ones, A. C.; Rushworth, S. A. Polyhedron 1990, 9, 1483.
CdS particles) was discarded. The toluene was pumped off under vacuum to give a yellow material which was washed with $\mathrm{MeOH}$ to remove any free TOPO. This solid readily redisperses in toluene to give bright yellow, stable, solutions containing capped CdS nanocrystallites. Syntheses using the other molecular precursors followed a similar procedure.

Size selective precipitation was performed by adding $\mathrm{MeOH}$ to a toluene sol ution containing the semi conductor nanocrystallites until turbidity was observed followed by centrifugation of the solid. This procedure was successively applied to the supernatant solutions obtained during the fractionation process until no optical absorption was detected.

CdS and CdSe nanocomposites: The following organic ligands were used for the surface derivatization of CdS and CdSe nanocrystallites: pyrazine, 2,2'-bipyrimidine, and 4,4'-dipyridyl. The system based on CdE nanocrystallites and 2,2'-bipyrimidine was studied in most detail. In a typical preparation a toluene solution $(10 \mathrm{~mL})$ containing $30 \mathrm{mg}$ of CdS/TOPO nanocrystallites was prepared; this solution was added to $70 \mathrm{~mL}$ of 2,2'-bipyrimidine $\left(0.035 \mathrm{~mol} \mathrm{dm}^{-3}\right)$ in hot toluene, and a yellow suspension formed. The mixture was then refluxed and/or stirred for $24 \mathrm{~h}$ under a nitrogen flow. The materials obtained were isolated by centrifugation and washed twice with toluene $(40 \mathrm{~mL})$; the solid was then redispersed in pyridine $(8 \mathrm{~mL})$, after which petroleum ether (32 $\mathrm{mL}$ ) was added and centrifugation was performed. This procedure was repeated. Finally the solid obtained was dried under vaccum giving, e.g., $26 \mathrm{mg}$ of CdS/bpm nanocomposite. The CdSe nanocomposites were prepared using the same procedure.

When pyrazine or 4,4'-dipyridyl were used a similar method was applied, but the formation of a suspension was not immediately observed when ligand and $\mathrm{CdE} / \mathrm{TOPO}$ nanocrystallites were mixed in toluene. The nanocomposites were then washed twice with toluene $(40 \mathrm{~mL})$.

2.3. Instruments and Characterization. Optical absorption and luminescence measurements: The solutions containing CdS and CdSe nanocrystallites were placed into silica cuvettes $(1 \mathrm{~cm})$ and characterized by optical absorption spectroscopy with a Philips PU 8710 spectrophotometer. The solvent (toluene or pyridine) was used as reference.

The luminescence results were obtained at room temperature on a Spex FluoroM ax instrument. The solutions analyzed were prepared as detailed above. The solid samples were prepared by dispersing the solid in pyridine, and a few drops were left on a glass substrate to evaporate the solvent at room temperature.

NMR spectroscopy: The proton NMR spectra were recorded on a Bruker 250 AM pulsed Fourier transform instrument using $\mathrm{C}_{6} \mathrm{D}_{6}$ as solvent.

Infrared spectroscopy: The IR spectra were recorded on a Research Series instrument (samples 1\% in Csl disks).

X-ray powder diffraction: X-ray powder diffraction patterns were measured using a Guinier camera and a Philips 1130 $\mathrm{X}$-ray generator (Cu K $\alpha$ radiation). Samples for X-ray diffraction were prepared by placing the powder onto adhesive tape.

Transmission electron mi croscopy: Samples for transmission electron microscopy were prepared by putting an aliquot of a toluene solution of the nanocrystalline material onto an amorphous carbon substrate supported on a copper grid and then allowing the solvent to evaporate at room temperature. A J EOL-J EM 1200 EX II scanning and transmission electron microscope operating at $100 \mathrm{kV}$ was used for TEM. The histograms were obtained by measuring the bigger distance of 300 particles, for each sample, in the TEM images.

The HRTEM images and transmission el ectron diffraction patterns were obtained using a J EOL-J EM-2010 electron microscope operating at $200 \mathrm{kV}$ or a J EOL-J EM-4000EX electron microscope operating at $400 \mathrm{kV}$. To index the diffraction patterns, the camera constant was determined using an aluminum standard.

Elemental analysis: Microanalysis were carried out by the Microanalytical Laboratories at Imperial College. EDAX measurements were performed on samples deposited on aluminum substrates and using a J EOL J SM 35CF scanning electron microscope operating at $25 \mathrm{kV}$. ICPAES analysis (Cd, 

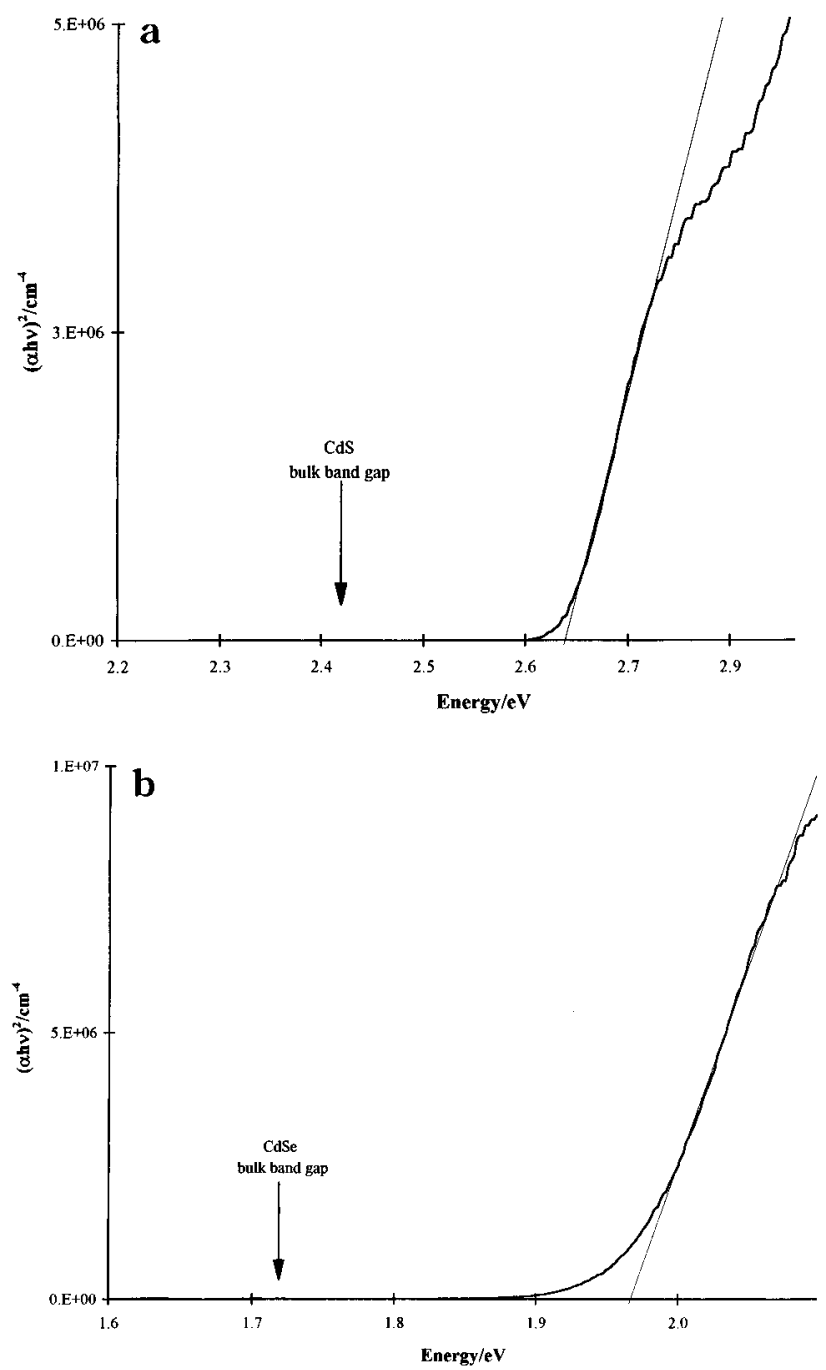

Figure 1. Optical absorption edges of nanodispersed CdS and CdSe in toluene fitted to a direct transition.

P) were recorded on a ARL instrument by Mr. B. Coles of the Geology Department, I mperial College.

\section{Results and Discussion}

3.1. Size-Dependent Optical Properties. It is well established that when the particle size of a semiconductor becomes comparable to the de Broglie wavelength of an electron (or hole), three-dimensional quantum confinement, imposed by the dimensions of the nanocrystal, occurs. ${ }^{1-9}$ The shift of the absorption edge of the semiconductor to higher energy provides experimental evidence for such quantum confinement and corresponds to an increase of the energy gap between the valence band and the conduction band of the semiconductor. Hexagonal macrocrystalline samples of $\mathrm{CdS}$ and CdSe have optical bandgaps at room temperature of $2.41 \mathrm{eV}(515 \mathrm{~nm})$ and $1.74 \mathrm{eV}(713 \mathrm{~nm})$, respectively. Figure 1 shows the fit of the absorption edge to a direct transition ${ }^{28}$ for $\mathrm{CdSe}$ and $\mathrm{CdS}$ prepared by thermolysis of $\left[\mathrm{MeCdE}{ }_{2} \mathrm{CN}\left(\mathrm{C}_{2} \mathrm{H}_{5}\right)_{2}\right]_{2}(\mathrm{E}=\mathrm{S}$, Se) in TOPO. In both cases the absorption band edge is blueshifted in relation to the bulk bandgap of the material. The blue-shifts observed in the spectra correspond to CdSe and CdS nanocrystals with dimensions comparable to the $\mathrm{CdE}$ bulk exciton radius.

(28) Pankove, J. I. Optical Processes in Semiconductors; Dover Publications Inc: : New York, 1970.
To narrow the size distribution, selective precipitation procedures were performed. Samples of CdS or CdSe nanocrystallites characterized by distinct size distributions were prepared. For example, Figure 2 shows the results for three selected fractions of CdS dispersed in toluene. The starting sample (A) has an absorption edge (2.65 eV) closer to the bulk bandgap of CdS than the fractions $\mathrm{B}(2.71 \mathrm{eV})$ or $\mathrm{C}(2.73 \mathrm{eV})$. The absorption edge of the nanodispersed CdS moves toward higher energy for the fractions obtained later in the process. As confirmed by the blue-shift observed in the spectra in Figure 2 the late fractions are richer in smaller particles. The TEM results in Figure 2 (typical TEM images and histograms) are in qualitative agreement with the optical measurements made on each sample. Fraction $C$ has a mean diameter (d) of $42 \AA \pm 7 \AA$, blueshift of $0.31 \mathrm{eV}(58 \mathrm{~nm})$; fraction $B(d=50 \AA \pm 8 \AA)$, blue-shift of $0.29 \mathrm{eV}(55 \mathrm{~nm})$; fraction $A(\mathrm{~d}=53 \pm 9 \AA)$ blue-shift of $0.23 \mathrm{eV}(44 \mathrm{~nm})$. The dispersion of the size distribution is also smaller for the later fractions $\left(\sigma_{C}<\right.$ $\sigma_{\mathrm{B}}<\sigma_{\mathrm{A}}$ ) which together with the smaller mean diameters explains why distinct excitonic peaks are observed in the optical spectra of fractions B and C. These absorption features correspond to the first electronic transition occurring in small CdS nanocrystallites. ${ }^{1-9}$ The wavelength at which the excitonic peaks are located is characteristic of CdS particles smaller than the bulk exciton and should be observed for materials close to monodispersed. ${ }^{29,30}$ The shifts observed for the excitonic peaks of the different fractions are relatively larger than the shifts observed at the absorption edges. The width of the absorption band ${ }^{4}$ is associated with the dispersion of the particles size (and vibrational coupling), which suggests that further size fractionations for samples A-C could narrow the size dispersion.

The results above are typical of CdS or CdSe nanocrystallites obtained from the thermal decomposition $\left(250{ }^{\circ} \mathrm{C}\right)$ of $\left[\mathrm{MeCdE}_{2} \mathrm{CNEt}_{2}\right]_{2}$. Some of the results using other single-molecule precursors and other synthetic conditions are summarized in Table 1 . In the case of $\mathrm{CdSe}$, the use of the $\left[\mathrm{Cd}\left(\mathrm{Se}_{2} \mathrm{CNEt}_{2}\right)_{2}\right]_{2}$ leads to the formation of some elemental Se as a solid phase, and its use was not investigated in detail. No significant differences were detected in the optical properties of CdS or $\mathrm{CdSe}$ when the neopentyl derivatives were used $\left(\left[\mathrm{NpCdE}_{2} \mathrm{CNEt}_{2}\right]_{2}\right)$. The absorption edge of CdS obtained from $\left[\mathrm{Cd}\left(\mathrm{S}_{2} \mathrm{CNEt}_{2}\right)_{2}\right]_{2}$ did show a slight red-shift as compared to CdS samples obtained from the other precursors. Nanocrystallites obtained in this case were in fact slightly bigger, and some agglomeration was found to have occurred by TEM measurements. It was found that the best procedure to prepare nanodispersed $\mathrm{CdE}$ with specific optical features was by recording the optical spectra and adjusting the temperature of reaction as required.

The use of low temperatures (Table 1) led to nanodispersed CdSe with optical features similar to those reported by Bawendi et al. for CdSe prepared at lower temperatures by the conventional TOPO method. ${ }^{16}$ The nanodispersed CdSe prepared at low temperature (120

(29) Spanhel, L.; Haase, M.; Weller, H.; Henglein, A. J . Am. Chem. Soc. 1987, 109, 5649.

(30) Fojtik, A.; Weller, H.; Koch, U.; Henglein, A. Ber. Bunsen-Ges. Phys. Chem. 1984, 88, 969. 

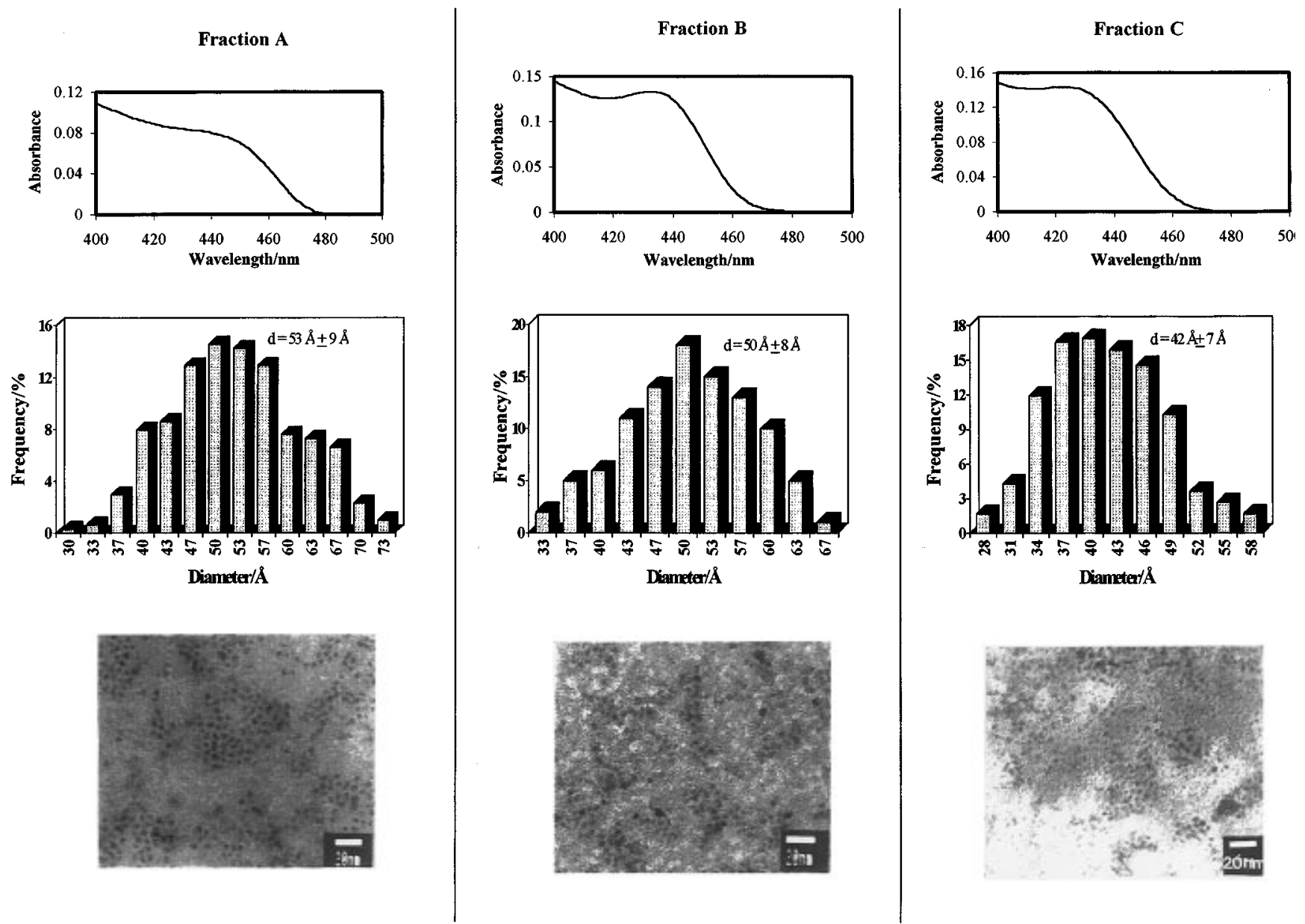

Figure 2. Optical absorption spectra; particle size histograms and TEM images (bar $=20 \mathrm{~nm}$ ) of size fractionated CdS nanocrystallites (fractions $A, B$, and $C$ ).

Table 1. Summary of Some Relevant Experimental Parameters in the Thermolysis of Single-Molecule Precursors

\begin{tabular}{|c|c|c|c|c|}
\hline precursor & $\underset{{ }^{\circ} \mathrm{C}}{\mathrm{temp} /}$ & $\begin{array}{l}\text { time/ } \\
\min \end{array}$ & product & comments \\
\hline$\left[\mathrm{Cd}\left(\mathrm{S}_{2} \mathrm{CNEt}_{2}\right)_{2}\right]_{2}$ & 250 & 30 & CdS hexagonal & yellow solid soluble in hydrocarbons; hexagonal CdS (X-ray diffraction) \\
\hline$\left[\mathrm{NpCdS}_{2} \mathrm{CNEt}_{2}\right]_{2}$ & 250 & 30 & CdS hexagonal & idem \\
\hline$\left[\mathrm{MeCdS}_{2} \mathrm{CNEt}_{2}\right]_{2}$ & 250 & 30 & CdS hexagonal & idem; see text for further characterization \\
\hline$\left[\mathrm{Cd}\left(\mathrm{S}_{2} \mathrm{CNEt}_{2}\right)_{2}\right]_{2}$ & 200 & 30 & CdS & $\begin{array}{l}\text { pale yellow solid soluble in hydrocarbons; nano-CdS with } \lambda_{\mathrm{exc}}=430 \mathrm{~nm} \\
\text { (optical measurements) }\end{array}$ \\
\hline$\left[\mathrm{Cd}\left(\mathrm{Se}_{2} \mathrm{CNEt}_{2}\right)_{2}\right]_{2}$ & 250 & 30 & CdSe hexagonal & $\begin{array}{l}\text { dark red solid soluble in hydrocarbons; grey insoluble solid; hexagonal } \\
\text { CdSe and hexagonal Se (X-ray diffraction) }\end{array}$ \\
\hline$\left[\mathrm{NpCdSe}_{2} \mathrm{CNEt}_{2}\right]_{2}$ & 250 & 30 & $\begin{array}{l}\text { Se hexagonal } \\
\text { CdSe hexagonal }\end{array}$ & $\begin{array}{l}\text { dark red solid soluble in hydrocarbons; grey insoluble solid; hexagonal CdSe } \\
\text { (X-ray diffraction) }\end{array}$ \\
\hline$\left[\mathrm{MeCdSe}_{2} \mathrm{CNEt}_{2}\right]_{2}$ & 250 & 30 & CdSe hexagonal & idem; see text for further characterization \\
\hline$\left[\mathrm{MeCdSe}_{2} \mathrm{CNEt}_{2}\right]_{2}$ & 200 & 30 & CdSe & soluble pale red solid; nano-CdSe with $\lambda_{\text {exc }}=546 \mathrm{~nm}$ (optical measurements) \\
\hline$\left[\mathrm{MeCdSe}_{2} \mathrm{CNEt}_{2}\right]_{2}$ & 180 & 40 & CdSe & soluble yellow/orange solid; nano-CdSe with $\lambda_{\mathrm{exc}}=503 \mathrm{~nm}$ (optical measurements) \\
\hline$\left[\mathrm{MeCdSe}_{2} \mathrm{CNEt}_{2}\right]_{2}$ & 120 & 30 & CdSe & soluble yellow solid; nano-CdSe with $\lambda_{\mathrm{exc}}=410 \mathrm{~nm}$ (optical measurements) \\
\hline
\end{tabular}

$\left.{ }^{\circ} \mathrm{C}\right)$ have a larger blue-shift in the excitonic peak which is consistent with a molecular-like species without a completely defined crystal structure. This type of small CdE clusters have been reported ${ }^{15,16,31-33}$ to gi ve opti cal maxima considerably blue-shifted from the typical bandgap of the semiconductor and are subject of intense research. ${ }^{32,33}$

The optical absorption edges observed for the spectra of nanodispersed CdS and CdSe give an estimate of

(31) Brennan, J . G.; Siegrist, T.; Carrol, P. J .; Stuczynski, S. M.; Brus, L. E.; Steigerwald, J . J . Am. Chem. Soc. 1989, 111, 4141.

(32) Herron, N.; Calabrese, J . C.; F arneth; W. E.; Wang, Y. Science 1993, 259, 1426.

(33) Vossmeyer, T.; Reck, G.; Katsikas, L.; Haupt, E. T. K.; Schulz, B.; Weller, H. Science 1995, 267, 1476. particles size/quantum effects. The large surface/ volume ratios typical of nanocrystalline materials mean that special attention should be paid to the study of the surface characteristics. The surface of a nanocrystalline material has a remarkable effect on its luminescence properties. ${ }^{16,17,29,30,34,35}$ Macrocrystalline CdS has a broad emission extending across the red region of the spectrum which has been related to the recombination of the charge carriers at defects. $29,30,34,35$ In CdS these defects consist of highly electronegative $\mathrm{S}^{2-}$ vacancies.

(34) Fisher, Ch. H.; Weller, H.; Katsikas, L.; Henglein, A. Langmuir 1989, 5, 429.

(35) Chestnoy, N.; Harris, T. D.; Hull, R.; Brus, L. E. J . Phys. Chem. 1986, 90, 3393. 
The $\mathrm{S}^{2-}$ vacancies act as deep traps for photogenerated electrons in the conduction band. It was suggested ${ }^{35}$ by Brus et al. that the red luminescence of $\mathrm{CdS}$ nanocrystallites results from the transfer of a trapped conduction electrons from $S^{2-}$ vacancies $\left(V_{S}{ }^{\circ}\right)$ to a surface molecular acceptor or to a preexisting trapped hole. In the present work the red emission of $\mathrm{CdE}$ is absent in the case of nano-CdSe and of diminished intensity in the case of nano-CdS in toluene. The emission spectrum was found to have a maximum at $472 \mathrm{~nm}(2.61 \mathrm{eV})$ for CdS and at $596 \mathrm{~nm}(2.08 \mathrm{eV})$ for CdSe, for excitations at 370 and $465 \mathrm{~nm}$, respectively. These features are close to the optical absorption edge of CdS and the excitonic peak of CdSe for the same samples, which suggests that near band-edge emission (shallow trap emission) is present. The band-edge emission is normally quenched by the deep trap emission due to the recombination of trapped charge carriers. The fluorescence experiments suggest that the nanocrystallites are capped by TOPO molecules, which fill surface defects as discussed above, in agreement with the reports of other authors. ${ }^{16-18}$

3.2. Structural Characterization. Nanocrystals of both CdS or CdSe have been reported to exist as either the cubic $c^{2,36,37}$ or hexagonal phase; ${ }^{16}$ however, a clear distinction between the two phases can be difficult if there is a lack of structural uniformity within the nanocrystallites. Bawendi et al. ${ }^{38}$ reported, on the basis of detailed modeling of X-ray powder patterns, that CdSe nanocrystallites, as synthesized in inversed micelles, referred to as cubic type CdSe, which are more likely to have a mixture of cubic and hexagonal domains.

Although the electrooptical properties of large semiconducting nanocrystallites differ from those of bulk materials, the crystalline structures are the same. ${ }^{4}$ X-ray powder diffraction of the CdS and CdSe nanocrystallites obtained in this study have patterns consistent with the hexagonal phases for both materials. However, the small particle size means that the X-ray patterns are broad; electron diffraction is in this case a valuable additional technique for characterization.

Electron diffraction and high resolution transmission electron microscopy (HRTEM) can give information on thestructure of the nanocrystallites. In Table 2 el ectron diffraction patterns for CdS and CdSe nanocrystallites are reported. The indexing of the observed lattice parameters to the hexagonal phases is reported confirming the predominance of the hexagonal phase for both CdS and CdSe. Figure 3a,b shows the HRTEM images of nanocrystalline CdS and CdSe, respectively, in which the lattice fringes are easily observed. When the particles are analyzed under the microscope the CdS consist in thinner nanocrystals compared to those of CdSe. The morphology for most of the CdS nanocrystallites is closer to thin plates rather than spheres, a result consistent with a preferential mode of arrangement of the CdS particles when they settle on the carbon coated copper grid.

(36) Salata, O. V.; Dobson, P. J .; Hull, P. J .; Hutchison, J . L. Thin Solid Films 1994, 251, 1.

(37) Kortan, A. R.; Hull, R.; Opila, R. L.; Bawendi, M. G.; Steigerwald, M. L.; Carroll, P. J .; Brus, L. E. J . Am. Chem. Soc. 1990, 112, 1327.

(38) Bawendi, M. G.; Kortan, A. R.; Steigerwald, M. L.; Brus, L. E. J . Chem. Phys. 1989, 91, 7282.
Table 2. Electron Diffraction Pattern of CdS and CdSe Nanocrystallites

\begin{tabular}{llcl}
\hline material & $\mathrm{d}(\mathrm{obsd}) / \AA$ & $\mathrm{d}($ ASTMS) $/ \AA \AA$ & $\mathrm{hkl}$ \\
\hline CdS & $3.584-3.212$ & 3.586 & 100 \\
& & 3.360 & 002 \\
& 2.433 & 3.164 & 101 \\
& 2.091 & 2.452 & 102 \\
& 1.912 & 2.071 & 110 \\
CdSe & 1.746 & 1.899 & 103 \\
& 1.389 & 1.763 & 112 \\
& $3.683-3.318$ & 1.399 & 203 \\
& & 3.72 & 100 \\
& 2.147 & 3.51 & 002 \\
& 1.825 & 3.290 & 101 \\
& 1.384 & 2.151 & 110 \\
& $1.235-1.180$ & 1.834 & 112 \\
& & 1.380 & 211 \\
& & 1.241 & 300 \\
& 1.016 & 1.222 & 301 \\
& & 1.206 & 213 \\
& & 1.022 & 311
\end{tabular}

a Hexagonal phase.

3.3. Synthesis and Characterization of CdEBased Nanocomposites. In the fabrication of luminescence devices based in semiconductor nanocrystallites, the control and manipulation of their surface composition is crucial. ${ }^{12,16,17,39-41}$ The band-edge luminescence shown by CdS and CdSe, as discussed before, is consistent with nanocrystallites having TOPO molecules attached to their surface. This suggests that by suitable surface derivatization it may be possible to enhance the luminescence properties of the materials for technological applications.

The characteristic IR bands of the capped $\mathrm{CdE}$ materials and the capping ligands are shown in Table 3. TOPO absorbs strongly at 1466 and $1146 \mathrm{~cm}^{-1}$. In particular the $\mathrm{P}=\mathrm{O}$ stretching is a useful probe for phosphine oxide molecules since the position of the characteristic band between 1320 and $1140 \mathrm{~cm}^{-1} \mathrm{de}-$ pends on the attached groups. ${ }^{42}$ A shift of $20 \mathrm{~cm}^{-1}$ for the $v(\mathrm{P}=\mathrm{O})$ band to lower wavenumber has been re ported ${ }^{17}$ by others for TOPO bound to CdSe, but this band was not observed in this work. Although of weak intensity the band of TOPO at $1466 \mathrm{~cm}^{-1}$ was observed for the CdS/TOPO (1468 $\left.\mathrm{cm}^{-1}\right)$ and CdSe/TOPO (1465 $\mathrm{cm}^{-1}$ ) nanocrystallites. It could be argued on the basis of these IR results that the TOPO present on the materials is not capping the semiconductor nanocrystallites surface. However, other experimental evidence supports the fact that sorption onto CdE surface has occurred, e.g., the high solubility of the nanocrystallites in hydrocarbon solvents; the observed strong band-edge emission of the nanodispersed materials and the presence of phosphorus, as detected by EDAX measurements made on samples after several washings (see Figure 4 for the CdS material). The shift observed by others on the $v(\mathrm{P}=\mathrm{O})$ frequency to lower values was explained by the binding of TOPO to $\mathrm{Cd}^{2+}$ sites on the CdSe nanocrystallites surface. ${ }^{17}$ One possible explanation for the

(39) Wang, Y.; Herron, N.; Mahler, W.; Suna, A. J . Opt. Soc. Am. B 1989, 6, 808.

(40) Mews, A.; Eychmuller, A.; Giersig, M.; Schooss, D.; Weller, H. J. Phys. Chem. 1994, 98, 934.

(41) Dabbousi, B. O.; Murray, C. B.; Rubner, M. F.; Bawendi, M. G. Chem. Mater. 1994, 6, 216.

(42) Colthup, N. B.; Daly, L. H.; Wiberley, S. E. Introduction to Infrared and Raman Spectroscopy, 3rd ed.; Academic Press Inc.: San Diego, 1990; p 364. 
Table 3. Selected Infrared Data (wavenumber/cm ${ }^{-1}$, Relative Intensity, Assignment) for CdS and CdSe Nanocrystallites with Derivatized Surface (TOPO and bpm) ${ }^{a}$

\begin{tabular}{|c|c|c|c|c|c|}
\hline TOPO & bpm & CdS/TOPO & $\mathrm{CdS} / \mathrm{bpm}$ & CdSe/TOPO & CdSe/bpm \\
\hline $\begin{array}{l}1466, \mathrm{~s}, \mathrm{shp} \\
\delta\left(\mathrm{CH}_{2}\right) \\
1146, \mathrm{vs}, \mathrm{shp} \\
v(\mathrm{P}=\mathrm{O})\end{array}$ & $\begin{array}{l}\text { 1562, vs, shp } \\
1555, \text { vs, shp } \\
1430, \text { s, shp } \\
1401, \text { vs, shp } \\
v \text { (ring) }\end{array}$ & $\begin{array}{l}\text { 1468, m, } \\
\text { shp } \\
\delta\left(\mathrm{CH}_{2}\right)\end{array}$ & $\begin{array}{l}1624, \mathrm{~s}, \mathrm{shp} \\
1603 \\
1569, \mathrm{~m}, \\
1444, \mathrm{~m}, \mathrm{shp} \\
1407, \mathrm{~m}, \mathrm{shp} \\
v \text { (ring) }\end{array}$ & $\begin{array}{l}1465, \mathrm{~m}, \mathrm{shp} \\
\delta\left(\mathrm{CH}_{2}\right)\end{array}$ & $\begin{array}{l}\text { 1623, m, shp } \\
1571, \mathrm{w} \\
1444, \mathrm{w} \\
1407, \mathrm{~m} \text {, shp } \\
v \text { (ring) }\end{array}$ \\
\hline
\end{tabular}

a $\delta$ : bending; $v$ : stretching; w: weak; m: medium; s: strong; vs: very strong; shp: sharp.

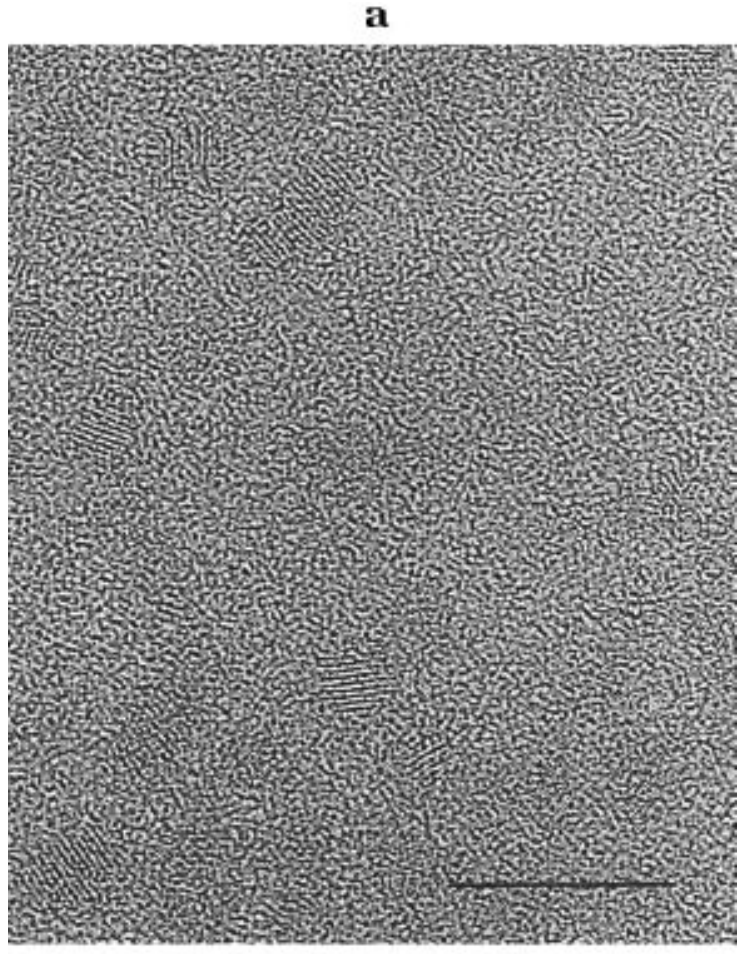

b

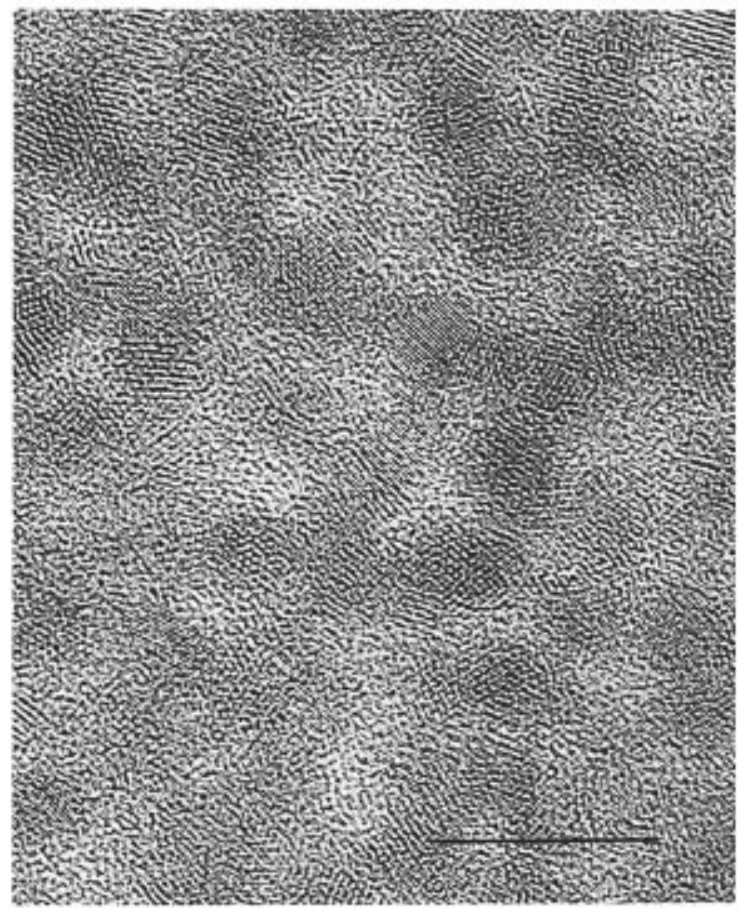

Figure 3. HRTEM images of (a) CdS and (b) CdSe nanocrystallites (bar = $10 \mathrm{~nm}$ ).

results obtained here could be related to the TOPO composition at the particles' surface being lower when

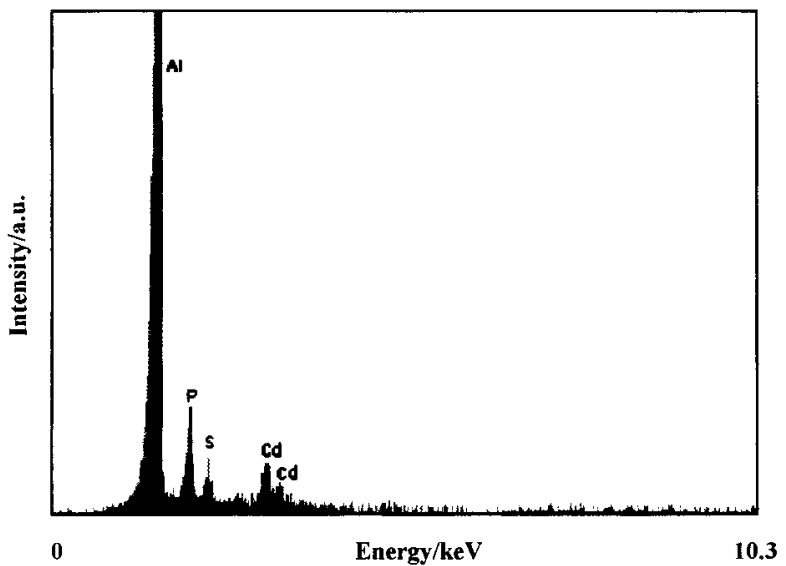

Figure 4. EDAX made on a CdS/TOPO sample over an aluminum substrate.

single-molecule precursors are used in comparison to the conventional method where an excess of $\mathrm{Me}_{2} \mathrm{Cd}$ was used. The ratio of $\mathrm{Cd} / \mathrm{E}$ atoms in the precursors used here is $1 / 4$, i.e., an excess of chalcogenide chemical species is present in relation to the metal, which could lead to a different surface composition for the $\mathrm{CdE}$ nanocrystallites which is sufficient to affect the $v(\mathrm{P}=\mathrm{O})$ mode in the IR spectra.

In a first attempt to prepare novel composite materials the ligand 2,2'-bipyrimi dine (bpm) was used (Figure $5)$. The choice of this amine was prompted by the facts that (i) the molecule bpm has two pairs of nitrogens with chelating properties and (ii) the pairs of $\mathrm{N}$ donor atoms are on opposite sides making bridging possible via delocal ized $\pi$ orbitals.

On mixing an optically clear solution containing $\mathrm{CdE}$ nanocrystallites with bpm in toluene a suspension forms, from which after reflux, on cooling to room temperature a solid can be isolated by centrifugation. The IR spectra of these solids have bands in the region of the ring stretching of bpm, and the TOPO vibrational modes $\left(\mathrm{CH}_{2}\right.$ bending and $\mathrm{P}=\mathrm{O}$ stretching) were not detected (Table 3). The frequencies for the bpm bands are observed at higher values than for the free ligand which may be associated with the coordination of the ligand to the surface of $\mathrm{CdE}$ nanocrystallites. The shifts observed for the ring modes of bpm are similar for CdSe and $\mathrm{CdS}$, suggesting the same type of bonding of bpm to both materials.

Other bridging molecules, such as pyrazine (pyr) and 4,4'-dipyridyl (dpy), can also be used to prepare CdE nanocomposites. The IR spectra of $\mathrm{CdE} / p y r$ and $\mathrm{CdE} /$ dpy nanocomposites show bands in the ring stretching region shifted to higher frequencies when compared to the free ligand (Table 4), which may be due to coordination to the nanocrystallites. Also the ring stretching vibrational bands were found to be in larger number in 

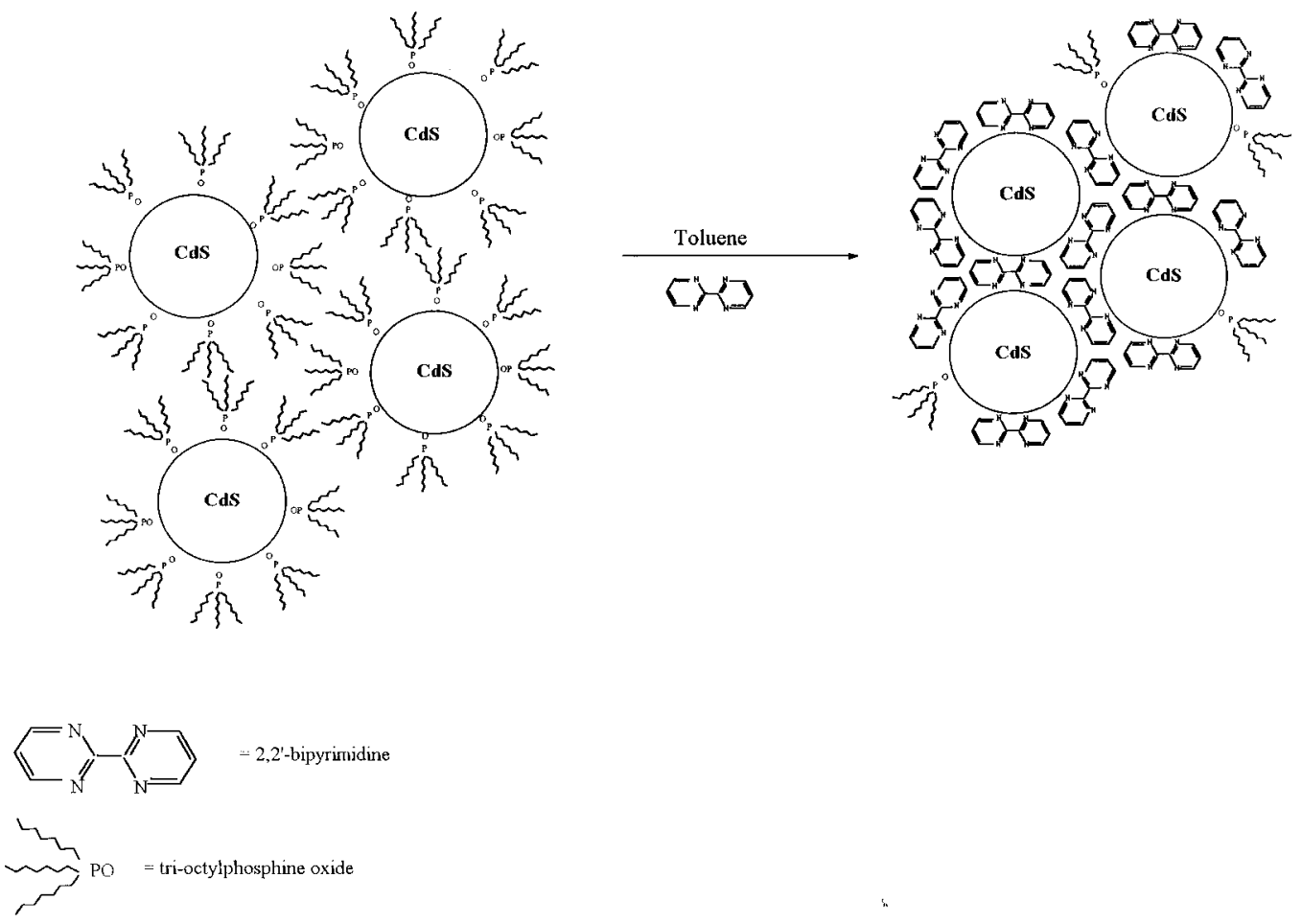

Figure 5. Proposed scheme for the formation of $\mathrm{CdS} / 2,2^{\prime}$-bipyrimidine nanocomposite (for sake of clearness the draw is not scaled).

Table 4. Selected Infrared Bands in the Ring Stretching Region for CdE/pyr and CdE/dpy Nanocompositesa

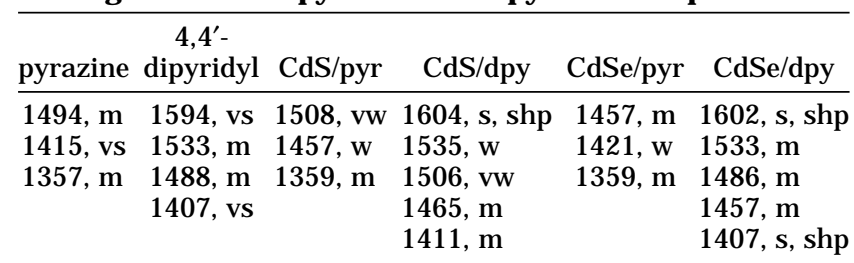

a a: data in wavenumbers $\left(\mathrm{cm}^{-1}\right)$. w: weak; m: medium; s: strong; vs: very strong; shp: sharp.

some of the nanocomposites (Tables 3 and 4), as compared to the free ligand, which can be associated to loss of symmetry in the coordinated ligand molecules.

The exact mechanism for the assembly of the CdE nanocrystallites is not clear. The results obtained from elemental analysis suggest that some TOPO molecules remain bound to the surface of the partides, even though the IR spectra did not show the characteristic $v(\mathrm{P}=\mathrm{O})$ band (Table 3). The content of $\mathrm{Cd}, \mathrm{N}$, and $\mathrm{P}$ found in the nanocomposite are from $\mathrm{CdE}$, bpm, and TOPO, respectively. In the CdS/bpm system $\left(\mathrm{d}_{\mathrm{Cds}} \approx 52\right.$ $\AA)$, for example, the empirical formula of (CdS $)_{4.7^{-}}$ $\mathrm{TOPO}_{0.42}$ bpm $_{0.35}$ was found for the nanocomposites obtained from stirring CdS/TOPO with bpm. The number of bpm molecules increases, in relation to the $\mathrm{CdS}$ and TOPO content, on reflux to give $(\mathrm{CdS})_{3.4} \mathrm{~T}$ $\mathrm{OPO}_{0.42} \mathrm{bpm}_{0.92}$. The empirical formula (CdS) $)_{5.4}(\mathrm{TOPO})_{0.67}$ was found for the initial nanoparticulates. These results suggest that although the bpm molecules did not replace completely TOPO they bind to the CdS surface and can assembly the semiconductor nanocrystallites into new materials ( $\mathrm{CdE} / \mathrm{bpm})$ as schematically proposed in Figure 5.

It is suggested that the bpm coordinates perpendicularly to the $\mathrm{CdE}$, through the lone pairs on the $\mathrm{N}$ atoms, on the basis of reported surface enhanced Raman studies using Ag sols. ${ }^{43}$ The elemental analysis results al so confirm that the TOPO molecules are firmly bound to the nanocrystallite surface. The percent of surface coverage for spherical CdS with $52 \AA$ in diameter, assuming that each capping molecule (TOPO or bpm) binds to a surface site, can be approximately calculated giving for each case: $24 \%$, (CdS $)_{5.4}(\mathrm{TOPO})_{0.67} ; 31 \%$, (CdS) $4.7 \mathrm{TOPO}_{0.42} \mathrm{bpm}_{0.35} ; 75 \%$, (CdS) $3.4 \mathrm{TOPO}_{0.42} \mathrm{bpm}_{0.92}$, i.e., a result strongly dependent on the synthetic conditions and the capping agent used. The surface coverage for the CdS/TOPO nanocrystallites is slightly lower than the value reported ${ }^{17}$ by other authors for $\mathrm{CdSe} / \mathrm{TOPO}$ (30-60\%, the percentage coverage increases as the particle size decreases) obtained from XPS measurements, though of the same order of magnitude. However the results reported here for the CdS/bpm nanocomposite show a higher percent coverage than that found by Alivisatos et al. for CdSe/TOPO nanocrystallites. ${ }^{17}$ There are several factors that affect the surface coverage of the nanocrystallites and may explain these differences. ${ }^{17}$ The nanocrystallites morphology and, especially, the steric hindrance of the capping ligands used in each case are obviously different. It should be kept in mind that these considerations were based on a simple calculation on the percentage of surface coverage for spherical and monodispersed CdS (52 ̊ diameter) and assuming that each bpm molecule are bound to a surface site $\left(\mathrm{Cd}^{2+}: \mathrm{S}^{2-}=1\right.$ at the surface), consequently the structure in Figure 5 should be seen as a simple view of the structure of the nanocomposite.

The new nanocomposite material consists of an inorganic core, CdS or CdSe, surrounded by an organic framework, TOPO and bpm (Figure 5). The solubility in toluene of capped CdS/bpm and CdSe/bpm materials decreases, but they are still sparingly soluble in pyri-

(43) Sbrana, G.; Neto, N.; Muniz-Miranda, M.; Nocentini, M. J . Phys. Chem. 1990, 94, 3706. 

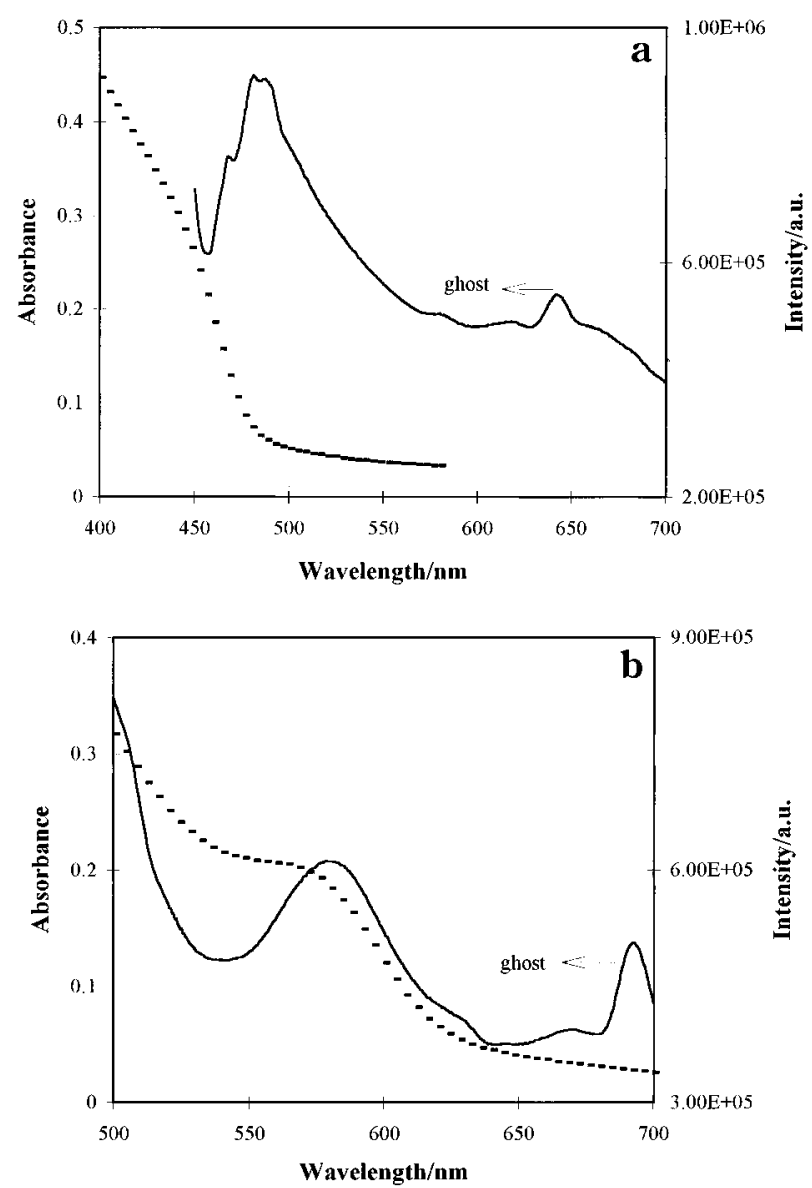

Figure 6. Optical absorption (dot line) and photoluminescence (solid line) spectra of (a) CdS/bpm ( $\lambda_{\text {exc }}=430 \mathrm{~nm}$ ) and (b) CdSe/ bpm $\left(\lambda_{\text {exc }}=465 \mathrm{~nm}\right)$, on glass substrate.

dine, presumably by competition for surface sites. Although exchange reactions at the surface of nanoparticles have been used to generate materials that could be connected by polimerization reactions ${ }^{44-47}$ or to bind compounds to Au surfaces, ${ }^{17}$ we believe this is the first time that a material has been prepared in which quantum dots are linked by a bridging molecular ligand.

The luminescence spectrum of a film consisting of the solid nanocomposite $\mathrm{CdS} / \mathrm{bpm}$ on a glass substrate shows as the main feature an emission maximum at 488 $\mathrm{nm}$ (Figure 6a) and at $580 \mathrm{~nm}$ for the anal ogue CdSe material (Figure 6b). The optical absorption spectra of these materials, dispersed in pyridine, are also shown in Figure 6. The absorption edge is located at $480 \mathrm{~nm}$ for the CdS/bpm optical spectrum, i.e., very cl ose to the

(44) Sankaran, V.; Yue, J .; Cohen, R. E.; Schrock, R. R.; Silbey, R. J. Chem. Mater. 1993, 5, 1133.

(45) Noglik, H.; Pietro, W. J. Chem. Mater. 1994, 6, 1593.

(46) Noglik, H.; Pietro, W. J . Chem. Mater. 1995, 7, 1333.

(47) Butterworth, M. D.; Armes, S. P.; Simpson, A. W. J . Chem. Soc., Chem. Commun. 1994, 2129. observed emission maximum (Figure 6a) and CdSe/bpm has an excitonic peak at $573 \mathrm{~nm}$. For both nanocomposites the emission maximum is observed at wavelengths slightly red-shifted from the optical absorption features. The results suggest that these materials show band-edge (or near band-edge) luminescence. Strong scattering from the glass substrate at wavelengths longer than $600 \mathrm{~nm}$ was clearly detected; the features observed at these wavel engths were also observed in the spectra of glass substrate. The broadening observed for the emission bands in the solids (Figure $6 a, b$ ) is probably associated with the film deposition method which was based on a simple evaporation of the solvent onto a glass substrate. The optical absorption spectra of $\mathrm{CdE} / \mathrm{bpm}$ in pyridine (Figure 6) are similar to the starting $\mathrm{CdE} / \mathrm{TOPO}$ nanoparticulates in tol uene (Figure 1). These results suggest that the $\mathrm{CdE} / \mathrm{bpm}$ nanocomposites retain the optical properties of the individual CdE nanoparticulates from which they were made. It is possible that ligand exchange with pyridine has also occurred however the pyridine bands were not detected in the IR spectrum. The CdE/pyr and CdE/dpy nanocomposites also show band-edge emission at the same wavelengths as the $\mathrm{CdE} / \mathrm{bpm}$ when the same $\mathrm{CdE} /$ TOPO material is used. These preliminary observations on novel composites suggest that defined complexes of nanocrystallites with suitable ligands can be formed. Although evidence is limited to analytical results and infrared spectroscopy, at present further studies on this new class of materials are in progress.

\section{Conclusions}

CdS and CdSe nanocrystallite's have been obtained by the thermal decomposition of dithiocarbamates and disel enocarbamates of cadmium in hot tri-n-octylphosphine oxide. The nanoparticulates clearly show quantum confinement and are close to monodispersed and crystalline. Using these CdS and CdSe nanocrystallites, a new class of nanocomposites can be obtained by derivatizing the nanocrystallites surface with bridging organic ligands such as 2,2'-bipyrimidine, 4,4'-di pyridyl, or pyrazine. These nanocomposites consist of an inorganic semiconducting core, CdS or CdSe, surrounded by an organic framework. The new nanocomposites still show, in the solid state, the band-edge emission observed for the starting semiconductor nanocrystallites.

Acknowledgment. We acknowledge Mr. K. Pell (QMW College) for the conventional TEM results, Miss R. Nayak and Dr. P. Dobson (Oxford University) for the HRTEM (400 kV) images, and Dr. Gary Rumbles (Imperial College) for helpful discussions about the luminescence results. T.T. acknowledges J NICT (Praxis XXI program) for a grant.

CM 960363R 